\title{
EUSTATHII THESSALONICENSIS
}

EXEGESIS

IN CANONEM IAMBICUM PENTECOSTALEM 


\section{SUPPLEMENTA BYZANTINA}

TEXTE UND UNTERSUCHUNGEN

HERAUSGEGEBEN VON

ATHANASIOS KAMBYLIS

BAND 10

DE GRUYTER 


\section{EUSTATHII THESSALONICENSIS \\ EXEGESIS}

IN CANONEM IAMBICUM PENTECOSTALEM

RECENSUERUNT INDICIBUSQUE INSTRUXERUNT

PAOLO CESARETTI - SILVIA RONCHEY 
Volume pubblicato con i seguenti contributi: fondi ex $60 \%$ MIURST (Paolo Cesaretti), fondi del Dipartimento di Lettere e Filosofia dell'Università degli Studi di Bergamo, fondi di ricerca del Dipartimento di Studi Umanistici dell’Università degli Studi di Roma Tre (Silvia Ronchey).

ISBN: 978-3-11-019521-7

e-ISBN (PDF) 978-3-11-022730-7

e-ISBN (EPUB) 978-3-11-039063-6

ISSN 1862-2496

Library of Congress Cataloging-in-Publication Data

A CIP catalog record for this book has been applied for at the Library of Congress.

Bibliografische Information Der Deutschen Nationalbibliothek.

Die Deutsche Nationalbibliothek verzeichnet diese Publikation in der Deutschen Nationalbibliografie; detaillierte bibliografische Daten sind im Internet über http://dnb.dnb.de abrufbar.

(C) 2014 Walter de Gruyter GmbH, Berlin/München/Boston

Satz: Dörlemann Satz GmbH \& Co. KG, Lemförde

Druck und buchbinderische Verarbeitung: Hubert \& Co. GmbH \& Co. KG, Göttingen $\infty$ Gedruckt auf säurefreiem Papier,

Printed in Germany

www.degruyter.com 